\title{
MS037.P05
}

\section{Netrin4 and laminin gamma-1 interacts via their N-terminal globular domains}

Trushar Patel $^{1}$, Matthew McDougall ${ }^{2}$, Raphael Reuten ${ }^{3}$, Denise Nikodemus ${ }^{4}$, Markus Meier ${ }^{2}$, Manuel Koch ${ }^{4}$, Joerg Stetefeld ${ }^{2}$

${ }^{1}$ Chemistry \& Biochemistry, University Of Lethbridge, Lethbridge, Canada, ${ }^{2}$ Department of Chemistry, University of Manitoba, Winnipeg, R3T 2N2, Canada, ${ }^{3}$ Biotech Research and Innovation Centre, University of Copenhagen, Copenhagen, DK-2200, Denmark, ${ }^{4}$ Institute for Dental Research and Oral Musculoskeletal Biology, Medical Faculty, University of Cologne, Cologne, 50931, Germany E-mail: trushar.patel@uleth.ca

Laminin is a heterotrimeric molecule composed of an alpha, a beta and a gamma chain. It interacts with various ligands at the extracellular matrix and plays a pivotal role in many biological processes. Netrin4, one of the four secreted netrins, specifically interacts with the laminin gamma-1 chain (Schneiders et al. 2007). We recently highlighted the biological significance of this complex using ex vivo and in vivo studies (Reuten et al. 2016). Furthermore, we also solved a structure of netrin 4 at $3.1 \AA$ that allowed us to reason why netrin 4 and our previously reported structure of netrin 1 (Grandin et al. 2016) interact with different binding partners. To implicate the structure of netrin $4 /$ laminin gamma- 1 complex and its biological functions, we employed a multidisciplinary approach where we combined the low-resolution shape information of netrin4/laminin gamma-1 complex with the high-resolution structure information of each component to obtain a highresolution model of the complex. This approach revealed that the $\mathrm{N}$-terminal globular domains of netrin4 and laminin gamma-1 are required for the interaction (Reuten et al. 2016). The interaction sites were validated using structure-guided mutations that provided detailed insights on the biological relevance of this complex (Reuten et al. 2016). The highresolution model of the netrin4/laminin gamma-1 complex was validated by comparing the experimentally derived hydrodynamic properties with those calculated from the structures.

1, Schneiders, F.I. and Maertens, B. et al. (2007) J. Biol. Chem. 282, 23750-23758

2, Reuten R. and Patel, T. R. et al. (2016) Nat. Commun. 7, 13515, 1-17

3, Grandin, M. and Meier, M. et al. (2016) Cancer Cell. 29, 175-185

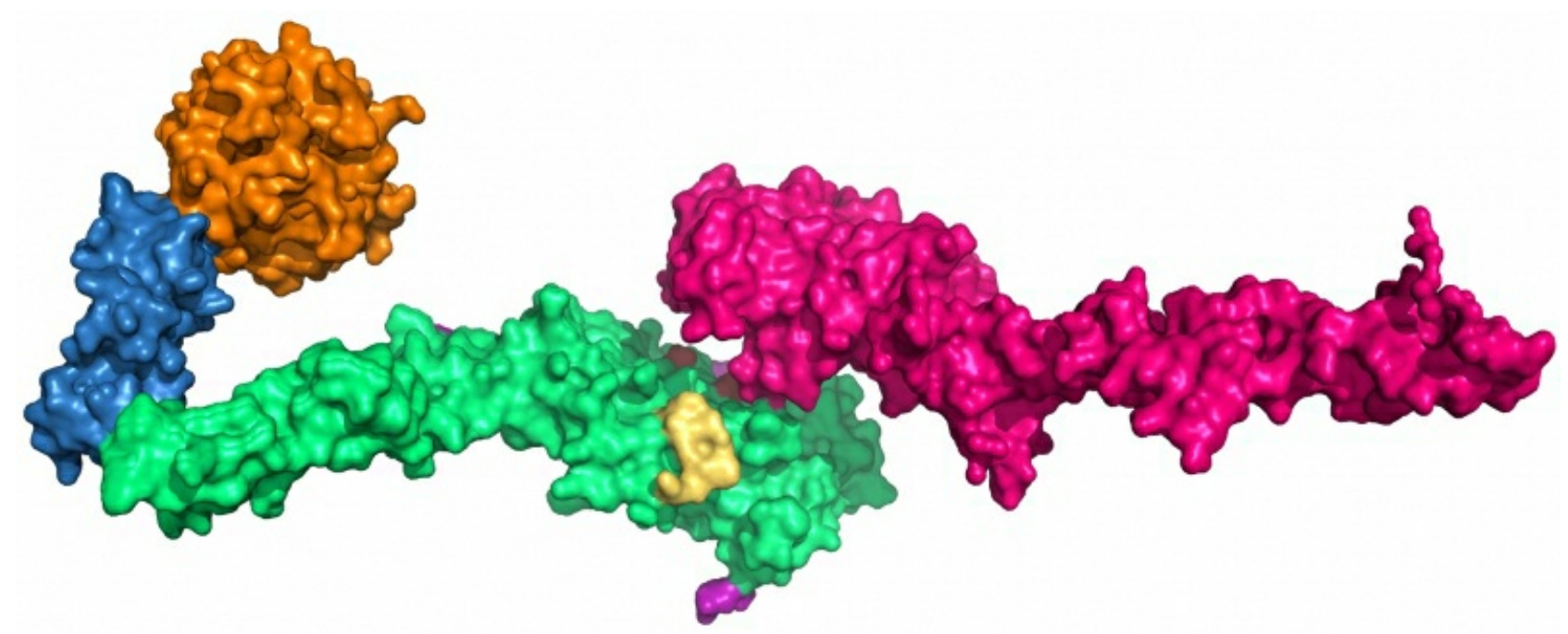

Keywords: extracellular matrix, solution X-ray scattering, X-ray crystallography 